\title{
THE STATUS-2004 OF THE KURCHATOV CENTER OF SR
}

V. Korchuganov, Budker INP SB RAS, Novosibirsk and KCSR RSC Kurchatov Institute, Moscow; M.Kovalchuk, S. Zheludeva, Shubnikov IC RAS, Moscow and KCSR RSC Kurchatov Institute, Moscow; Yu. Krylov, V. Kvardakov, D. Odintsov, V. Ushkov, A. Valentinov, Yu. Yupinov, KCSR RSC Kurchatov Institute, Moscow

\section{Abstract}

Kurchatov Synchrotron Radiation Source (KCSR) began the work as a first dedicated synchrotron radiation facility in Russia in 1999. The facility includes $450 \mathrm{MeV}$ SIBERIA-1 and $2.5 \mathrm{GeV}$ SIBERIA-2 storage rings. It is intended for experiments in the range of SR from VUV up to hard X-ray. Large progress was achieved in increasing stored current at SIBERIA-2 during last year. The report describes the current work and the plans on the storage rings. It informs about achieved consumer parameters of an electron beam and status of SR stations.

\section{INTRODUCTION}

At present, the Kurchatov SR source operates close to the design parameters. Table 1 presents the main features of the acting optical structure of the storage rings Siberia-1 and Siberia-2.

Table 1: Main parameters of Siberia-1 and Siberia-2 rings

\begin{tabular}{|l|l|l|}
\hline Parameter & Siberia-2 & Siberia-1 \\
\hline Energy & $2.5 \mathrm{GeV}$ & $0.45 \mathrm{GeV}$ \\
\hline Circumference & $124.13 \mathrm{~m}$ & $8.68 \mathrm{~m}$ \\
\hline Optical structure & Modified DBA & FODO \\
\hline Superperiods & 6 & 1 \\
\hline Bet. tunes: $v_{\mathrm{x}}, v_{\mathrm{y}}$ & $7.772 ; 6.692$ & $0.793 ; 0.895$ \\
\hline Mom.compaction & 0.0104 & 1.64 \\
\hline Damping $x, y, s, m s$ & $3.04 ; 3.17 ; 1.49$ & $7.15 ; 7.15 ; 3.57$ \\
\hline Hor. emittance & $78-98 \mathrm{~nm}-\mathrm{rad}$ & $880 \mathrm{~nm}-\mathrm{rad}$ \\
\hline RF harmonic & 75 & 1 \\
\hline Energy spread & 0.000953 & 0.00034 \\
\hline Dipole field: $\mathrm{B}_{\mathrm{y}}$ & $1.7 \mathrm{~T}$ & $1.5 \mathrm{~T}$ \\
\hline ID space & $\begin{array}{l}2 \times 3 \mathrm{~m}(\eta=0) \\
5 \mathrm{x} 3.2 \mathrm{~m}(\eta \neq 0)\end{array}$ & - \\
\hline $\begin{array}{l}\text { Bunch length: } \sigma_{\mathrm{s}} \\
\text { (without IDs) }\end{array}$ & $1.84 \mathrm{~cm}$ & $30 \mathrm{~cm}$ \\
\hline SR pulse duration & $0.14 \mathrm{~ns} \mathrm{FWHM}$ & $2.35 \mathrm{~ns} \mathrm{FWHM}$ \\
\hline SR pulse spacing & $5.5-414 \mathrm{~ns}$ & $28.9 \mathrm{~ns}$ \\
\hline Current & $\begin{array}{l}100-150 \mathrm{~mA} \\
(\mathrm{multibunch})\end{array}$ & $\begin{array}{l}100-300 \mathrm{~mA} \\
(\text { single bunch) }\end{array}$ \\
\hline $\begin{array}{l}\text { Life time (100 mA, } \\
\text { coupling 1\%), hrs }\end{array}$ & $\begin{array}{l}10-14 \\
(\mathrm{multibunch})\end{array}$ & $\begin{array}{l}1.5(\mathrm{single} \\
\text { bunch) }\end{array}$ \\
\hline
\end{tabular}

\section{WORK WITH ELECTRON BEAM}

\section{Electron storage}

As rule the work on Siberia-2 SR goes in the multi- bunch regime with a current of $100-120 \mathrm{~mA}$ by filling of $1 / 2$ to $1 / 3$ of the ring ( 25 to 37 bunches).

The synchrotron oscillations collective modes appear after injection of first four or five bunches. Nevertheless we have realized that it is possible to store the electrons in all 75 bunches without specially made empty gap widely used to prevent ion trapping (see Fig.1a).

The energy ramping of the electrons with current in many bunches exceeding $150 \mathrm{~mA}$ is characterized by both big synchrotron motion in coherent modes and losses of the beam part. The losses depend on the number of bunches and modulate the particles numbers in bunches correspondingly with the synchrotron mode number (see Fig.1b).

a)

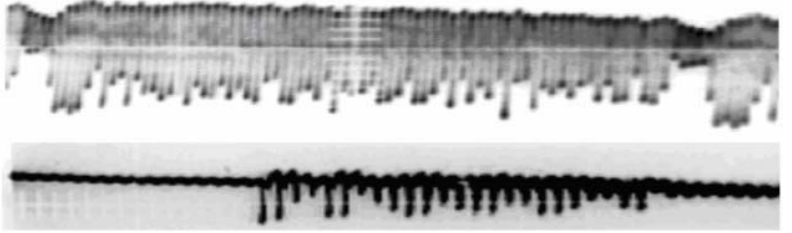

Figure 1a: The typical picture of the filling at the injection. Figure1b: The modulation due to collective mode instability losses during an energy ramping.

In single bunch mode the storage is accompanied by the bunch lengthening and microwave instability leading to the increasing of energy spread. In result the enlargement of the bunch was stopping the storage near $72 \mathrm{~mA}$ due to the losses of the particles as a consequence of the energy acceptance limitation. The maximum single bunch current accelerated up to $2.5 \mathrm{GeV}$ was about $30-35$ $\mathrm{mA}$ and was again limited by the coherent synchrotron oscillations, which are strong when ramping the energy within $0.45-1 \mathrm{GeV}$.

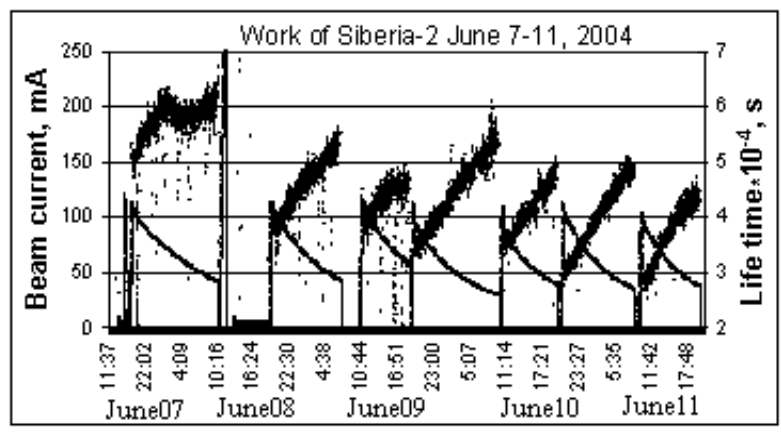

Figure 2: Work of Siberia-2 in June 7-11, 2004.

During routine operation on SR experiments the electron storage and acceleration regimes are reproduced 
in a stable way. Figure 2 shows the dependence of the beam current and the life time versus the time at $2.5 \mathrm{GeV}$ during the runs in June 07-11, 2004. Now the maximum currents achieved are: $300 \mathrm{~mA}(450 \mathrm{MeV})$ for Siberia-1 in a single bunch mode; $270 \mathrm{~mA}(450 \mathrm{MeV})$ and $150 \mathrm{~mA}$ $(2.5 \mathrm{GeV})$ for Siberia-2 in multibunch mode.

\section{Vacuum}

During last year the vacuum system of Siberia-2 was opened twice. In May 27, 2003, aluminum vacuum chamber in one of the BMs was replaced on new one in order to remove a vertical cutting of the SR beam. Next opening was made in February 2004 in order to change the vacuum insulator of the inflector. In both cases the vacuum chamber was degassed with an SR beam during two week without warming it up.

With the increasing of the electron current the serious problem became so called "hot" points arisen inside Siberia-2 vacuum tube due to SR parasite hit, which damaged the vacuum and the life time. Five such "hot" points with the sizes about $1-2 \mathrm{~mm}^{2}$ were found and three of them were removed. After February 2004 the vacuum condition was sharply improved and the life time was increased in a factor 2. After the spray of the titanium films in the magneto-discharge pumps around the ring the life time has exceeded 14 hours at $100 \mathrm{~mA}$ in the beginning of the week. But some degradation of the life time is observed to the end of the week run, perhaps due to working photon SR beam lines (see Fig.2).

Till now the life time is generally defined by the SR stimulated gas desorption. In fact Fig. 3 represents near linear behaviour of the product of an electron beam current and a life time versus total SR doze collected by the vacuum chamber from the beginning of work.

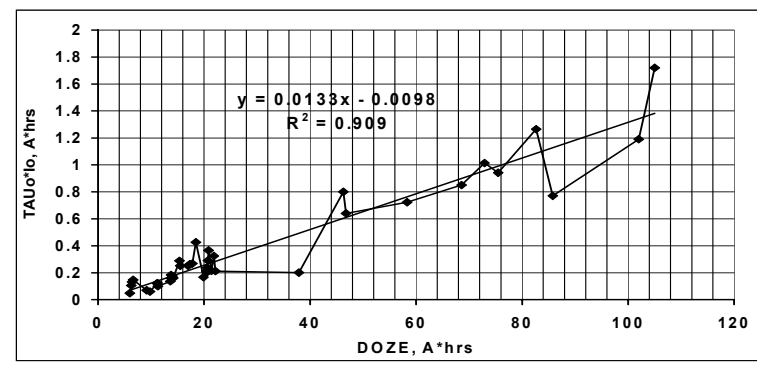

Figure 3: Dependence of the beam current and the life time product on the collected doze at $2.5 \mathrm{GeV}$.

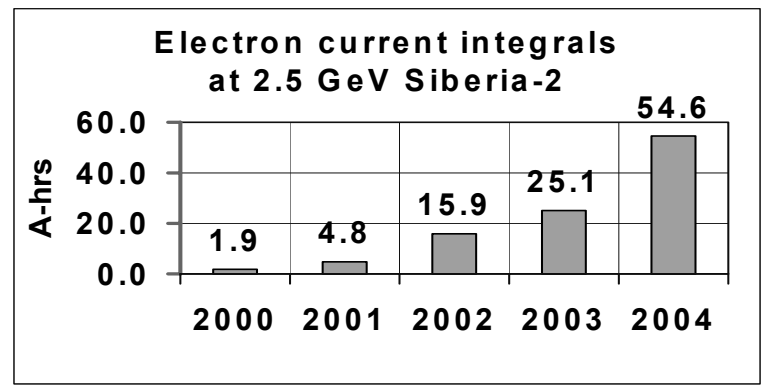

Figure 4: Annual dozes at Siberia-2.
Intensity of works with SR increased in 2004. As a result a total integral of current overcame $100 \mathrm{~A}^{*} \mathrm{~h}$ on June 8, 2004 and the doze of one half of 2004 is more than summarized doze of the 2000-2003 years (see Fig.4).

\section{COD CORRECTION AT SIBERIA-2}

Electron closed orbit distortion (COD) is measured by 24 pick-up stations with 10 micron accuracy at SIBERIA-2 storage ring. Correction system allows to achieve standard values of COD (at azimuths of BPMs) which are close to $\sigma_{\mathrm{z}}=0.2 \mathrm{~mm}$ and $\sigma_{\mathrm{x}}=0.7 \mathrm{~mm}$. To find COD at the 72 quadrupole lenses azimuths we also use the method of their gradient changes with the correction coils existing on quadrupole lenses poles. From this method the transversal relative distances between neighboring BPMs and quadrupole lenses centers are found and taken into account to improve COD control.

\section{PHOTON BEAMS STABILIZATION}

The photon beams of Siberia-2 are slowly moving vertically during many hours $(\sim 70 \mathrm{hrs})$ at $2.5 \mathrm{GeV}$ due to the temperature distribution change around the storage ring. Total SR beam shift during one-week operation was reaching 2-3 $\mathrm{mm}$ at a distance $15-20 \mathrm{~m}$. But after two days of the operation break the elements are coming back into initial positions. Now the feed-back system was made to correct SR beam position on two SR beam lines. It includes luminescent screens, TV-cameras, computer code with a space resolution of $2-3 \mathrm{mkm}$ and the control of 3 magnetic correctors, which create the local orbit distortion in vertical plane. The turned-on feed-back demonstrates that SR beam drift is damped with a precision of \pm 4-5 mkm during long time runs (see Fig.5).

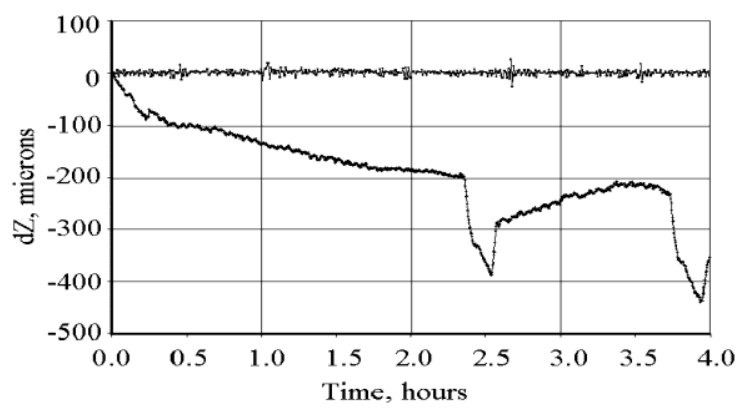

Figure 5: SR beam vertical position at precise X-Ray optics station with feed-back system turned-on and turned-off.

\section{SR BEAMS}

The Siberia-2 SR extraction scheme is the same for each of 12 one half of superperiods [2]. There are 39 SR extraction channels in total. Those include: 24 channels with SR from the bending magnets with the characteristic wavelength $\lambda_{\mathrm{c}}=1.75 \AA$ and horizontal angles $\pm 5 \mathrm{mrad}$; 10 SR channels with $\lambda_{\mathrm{c}}=(0.25-0.4) \AA$ from the two SCMP wigglers; 5 channels from the undulators with 
the fundamental wavelength $\lambda_{\text {fund }}=(20-1.7) \AA$ and multipole low field wigglers.

The works of SR stations at Siberia-2 are now performed with SR from the bending magnets over the photon energy range of 4 to $40 \mathrm{keV}$ and with $\left(10^{13}-10^{11}\right)$ phot $/ \mathrm{s} / 0.1 \% \mathrm{BW}$ fluxes. The SR source is operated regularly in accordance with the schedule. So, usually 7-8 12-hour shifts are allocated each week for work with the beam on the Siberia-2 ring. Figures 6, 7 shows diagrams of user time during last years dated on June 2004.

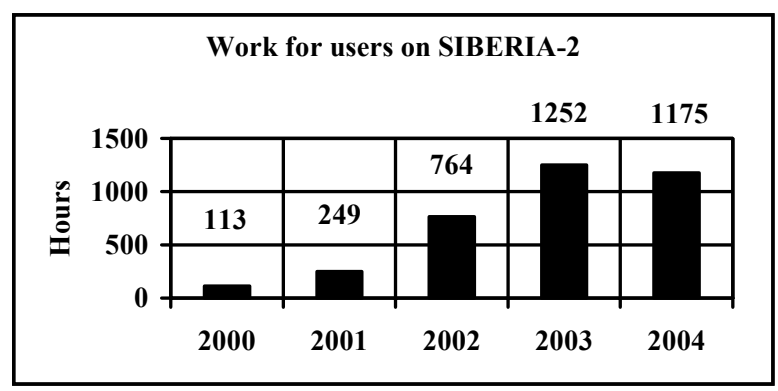

Figure 6: Siberia-2 as SR Source.

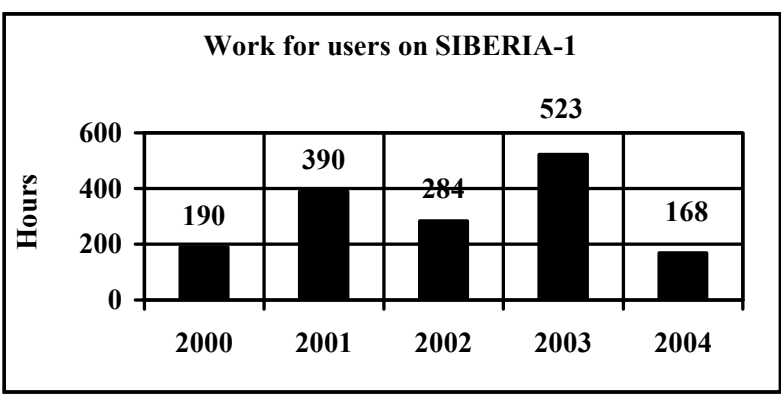

Figure 7: Siberia-1 as SR source.

\section{EXPERIMENTAL STATIONS}

Nine experimental stations are in operation at the X-ray experimental hall of Siberia-2 [3], which are dedicated for: protein crystallography, precise X-ray optics, X-ray crystallography and material science, medical and industrial diagnostics, LIGA, EXAFS, small angle scattering, time-resolved small-angle diffraction, X-ray luminescence. Three experimental stations are in operation at the VUV experimental hall for: photoelectron spectroscopy, VUV luminescence and optical investigations of solids.

The main directions of the scientific program include the structure diagnostics of an atomic level space resolution of organic and inorganic objects; protein crystallography and biotechnology, nanotechnology and nanodiagnostics; material science, X-ray optics, new methods for medical diagnostics, industrial nondestructive control; deep X-ray lithography et cetera.

Kurchatov Synchrotron Radiation Center is open for cooperation with domestic and foreign users of synchrotron radiation.

\section{PLANS ON SIBERIA-2}

The main task of future efforts is to increase intensity and life time of SR beams in Siberia-2 as well as to decrease electron beams emittances as a results of work with new magnetic optics of the ring in order to augment SR brightness [2].

\section{Optics with $66 \mathrm{~nm}$-rad horizontal emittance}

The main distinction between this structure and the base one is a large change of betatron frequencies: $v_{\mathrm{x}}=7.85 \quad v_{\mathrm{y}}=3.79$. The natural chromaticities stay relatively small: $\zeta_{\mathrm{x}}=-15.4, \zeta_{\mathrm{y}}=-11.7$, the calculated dynamical apertures are as such as $-20 \mathrm{~mm}<\mathrm{DA}_{\mathrm{x}}<29$ $\mathrm{mm},-24 \mathrm{~mm}<\mathrm{DA}_{\mathrm{y}}<24 \mathrm{~mm}$. The structure "66nmrad"do not require a special modification of the "iron".

\section{Optics with a $18 \mathrm{~nm}$-rad horizontal emittance}

According to calculations in the case of non-zero dispersion in all sections of Siberia-2, a structure with 18 nm-rad horizontal emittance (at an energy of $2.5 \mathrm{GeV}$ ), betatron tunes $\left(v_{\mathrm{x}}=9.708, v_{\mathrm{y}}=5.623\right)$ and chromaticities $\left(\zeta_{\mathrm{x}}=-21.4, \zeta_{\mathrm{y}}=-19.7\right)$, dynamical apertures $(-19 \mathrm{~mm}<$ $\left.\mathrm{DA}_{\mathrm{x}}<25 \mathrm{~mm},-12 \mathrm{~mm}<\mathrm{DA}_{\mathrm{y}}<12 \mathrm{~mm}\right)$ seems to be quite achievable at the present variability of parameters of magnetic elements. Such optics makes it possible to obtain a diffraction-limited radiation with rather shot wavelength. For instance, when working at electron energy about $1.33 \mathrm{GeV}$ horizontal emittance equals 4.8 $\mathrm{nm}$-rad. Then a diffraction-limited radiation in the vertical direction is reached at a wavelength of $\lambda_{\text {fund }}=6 \AA$ from the mini-undulator with space period of $7 \mathrm{~mm}$. But the "18 nm-rad" structure implies a modernization of the injection system and an optimization of the sextupole magnets positions on the ring.

\section{Installation of wigglers}

Activity in the protein crystallography and in material science is linked with installation of multipole superconducting wiggler with a field changed from 3 to $7.5 \mathrm{~T}$, photon flux up to $\left(10^{14}-10^{12}\right)$ phot./s/0.1\%BW and photon energies up to $200 \mathrm{keV}$.

\section{REFERENCES}

[1] V.V.Anashin et al., Nucl.Instrum.Meth., A282, p.369374,(1989).

[2] S.T.Belyaev, V.N.Korchuganov, M.V.Kovalchuk et al., "Status of Kurchatov SR Source", Proceedings of XVIII conf. on charge particle accelerators, 2002, Obninsk, Russia .

[3] M.V.Kovalchuk, V.V.Kvardakov, V.N.Korchuganov, S.I.Zheludeva,"Kurchatov Synchrotron Radiation Center", The 5-th Asian Synchrotron Radiation Forum in Saga, March 16-17 2004, Tosu, Saga, Japan, Extended Abstract, p. 77-80. 